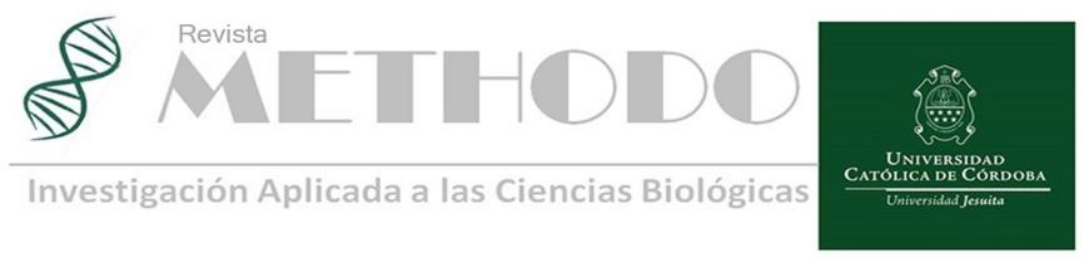

BIOESTADISTICA Y METODOLOGIA APLICADA Rev.Methodo 2020;5 (3):108-112 https://doi.org/10.22529/me.2020.5(3)06 Recibido 24 Oct. 2019 | Aceptado 18 May. 2019 | Publicado 03 Jul. 2020

\title{
El uso de glucocorticoides en neumonía grave adquirida de la comunidad
}

\section{Use of glucocorticoids in severe community acquired pneumonia}

Graciela Alegre $^{1}$, Augusto Buffa ${ }^{2}$, Micaela Y Colussi ${ }^{2}$, Candelaria Gimenez ${ }^{2}$, Sofia Manzanares ${ }^{2}$, Franca Monferini $^{2}$, Josefina Paschini ${ }^{2}$

1Universidad Católica de Córdoba. Facultad de Ciencias de la Salud. Cátedra de Inmunología. Clínica Universitaria Reina Fabiola, Servicio de Alergia e Inmunología.

2Universidad Católica de Córdoba. Facultad de Ciencias de la Salud. Estudiantes de sexto año, Carrera de Medicina.

Correspondencia: Graciela Alegre. Servicio de Alergia e Inmunología- Clínica Universitaria Reina Fabiola. Oncativo 1248 -X5004FHP- Córdoba, Argentina. Email: gracielaalegre@curf.ucc.edu.ar

\section{Resumen}

INTRODUCCION: La neumonía es la inflamación del parénquima pulmonar debida a un agente infeccioso. Cuando afecta a la población no ingresada en un hospital, se denomina neumonía adquirida en la comunidad (NAC). La NAC grave suele representar entre el 3 y $18 \%$ de las NAC hospitalizadas con una mortalidad entre el 21 y 54\%. Para evaluar la gravedad de la NAC se utilizan los criterios PSI (Pneumonia severity Index). Los corticoesteroides son hormonas esteroideas, participan en diferentes procesos regulando la inflamación, el sistema inmunitario, así como la respuesta del organismo al estrés, etc. Los corticoides sintéticos y semisintéticos se utilizan en numerosos procesos patológicos.

METODOLOGÍA: Desde la exposición de un escenario clínico, se planteó una pregunta estructurada para luego realizar una búsqueda bibliográfica, con el objetivo de dar respuesta a la pregunta sobre si el uso de corticoterapia adyuvante en pacientes con NAC grave disminuye la mortalidad y la necesidad de asistencia respiratoria mecánica.

Se realizó una búsqueda en PubMed y se seleccionó el artículo: "Efficacy and safety of glucocorticoids in the treatment of severe community-acquired pneumonia"

ANALISIS DEL ARTICULO: Se realizó un análisis definiendo los valores de riesgo absoluto, riesgo relativo y el número necesario a tratar del grupo problema y grupo control tomando en cuenta las variables mortalidad y necesidad de asistencia respiratoria mecánica. Por otro lado, se determinó el odds ratio con su respectivo intervalo de confianza del $95 \%$.

RECOMENDACIÓN FINAL: se recomienda el uso de corticoterapia adyuvante como tratamiento contra la NAC grave.

Palabras claves: Neumonía grave adquirida de la comunidad, glucocorticoides.

\section{Abstract}

INTRODUCTION: Pneumonia is the inflammation of lung parenchyma due to an infectious agent. When it affects population not admitted in a hospital, it is defined as community acquired pneumonia (CAP). Severe CAP represents between $3 \%$ and $18 \%$ of CAP hospitalizations, with a mortality ranging from $21 \%$

Revista Methodo: Investigación Aplicada a las Ciencias Biológicas. Facultad de Medicina Universidad Católica de Córdoba. Jacinto Ríos 571 Bo Gral. Paz. X5004FXS. Córdoba. Argentina. Tel.: (54) 3514517299 / Correo: methodo@ucc.edu.ar / Web: methodo.ucc.edu.ar| BIOESTADISTICA Y METODOLOGIA APLICADA Rev.Methodo 2020;5 (3):108-112. 
Alegre G, Buffa A, Colussi M Y, Giménez C, Manzanares S, Monferini F, Paschini J. El uso de glucocorticoides en neumonía grave adquirida de la comunidad.

to $54 \%$. To assess the severity of CAP, pneumonia severity index (PSI) criteria are used. Corticosteroids are steroid hormones that participate in different processes, regulating inflammation, the inmune system, as well as the body's response to stress. Synthetic and semi-synthetic corticosteroids are used in many pathological processes.

METHODOLOGY: From the presentation of a clinical scenario, a structured question was made and a bibliographic search was carried out, with the aim of answering the question about whether the use of adjuvant corticosteroid therapy in patients with severe CAP reduces mortality and the need of mechanical ventilation or not.

A PubMed search was performed and the article: "Efficacy and safety of glucocorticoids in the treatment of severe community-acquired pneumonia" was selected.

ARTICLE ANALYSIS: A secondary analysis of data was performed to define values for absolute risk, relative risk and number necessary to treat of the problem group and control group, considering the variables mortality and need of mechanical ventilation. The odds ratio was determined with its respective $95 \%$ confidence interval.

FINAL RECOMMENDATION: the use of adjuvant corticotherapy is recommended as a treatment against severe CAP.

Keywords: Community acquired severe pneumonia, glucocorticoids.

\section{Escenario clínico}

Paciente femenino de 66 años de edad consulta al servicio de emergencia acompañada por un familiar por presentar confusión de 6 hs de evolución. Como antecedentes, hace 48 hs se encuentra con fiebre de $40^{\circ} \mathrm{C}$, tos con expectoración, taquipnea y disnea. Al examen físico se constata una frecuencia respiratoria de 32 respiraciones por minuto, una frecuencia cardiaca de 125 latidos por minuto y a la auscultación se constata disminución de murmullo vesicular en campo medio y base pulmonar derecha y estertores crepitantes en el mismo lugar, saturando oxígeno a aire ambiente $90 \%$. Se realiza una telerradiografía de tórax donde se visualiza imagen de condensación en lóbulo inferior derecho. Luego de realizar estudios de laboratorio y teniendo en cuenta el índice de severidad para neumonía (PSI, Pneumonia Severity Index), la paciente se clasifica como de alto riesgo, por lo que se decide su internación en sala de cuidados intermedios para tratamiento y control clínico estricto. El médico a cargo de recibir a la paciente en la unidad de cuidados intermedios se cuestiona sobre la posibilidad de agregar tratamiento adyuvante con corticoterapia, ya que un colega le había comentado que en algunos centros médicos lo estaban haciendo.

\section{Pregunta estructurada}

¿El uso de corticoides por vía sistémica (intervención) en adultos que padecen neumonía grave adquirida de la comunidad (pacientes), disminuye el riesgo de mortalidad y el uso de asistencia respiratoria mecánica (ARM) (resultado)?

\section{Contexto clínico}

La neumonía es la inflamación del parénquima pulmonar debida a un agente infeccioso. Cuando afecta a la población no ingresada en un hospital, se denomina neumonía adquirida en la comunidad (NAC) o extrahospitalaria ${ }^{1}$ La incidencia de la enfermedad aumenta en pacientes en edades extremas de la vida, en varones y en invierno. También en pacientes con enfermedades respiratorias crónicas, HIV y en pacientes con hepatopatía crónica y diabetes. La neumonía es la causa más frecuente de muerte de origen infeccioso. Se hospitaliza alrededor del $40-60 \%$ de los pacientes con neumonía extrahospitalaria $^{1}$. La NAC grave suele representar entre el 3 y el $18 \%$ de las NAC hospitalizadas con una mortalidad que oscila entre 21 y el $54 \%$,3.

La presentación clínica de la NAC varía desde fiebre y tos productiva a distrés respiratorio y sepsis, en casos severos. La etiología microbiológica más frecuente de la NAC es la bacteriana, por Streptococcus pneumoniae y los virus respiratorios (Influenza, Parainfluenza, Virus Respiratorio Sincicial, Adenovirus). Sin embargo, existen otras bacterias que pueden desarrollar infección, como Haemophilus influenzae, Moraxella catarrhalis, Staphylococcus aureus, Mycoplasma pneumoniae, Chlamydia pneumoniae ${ }^{4}$.

Para evaluar la gravedad de la NAC se utilizan los criterios PSI (Pneumonia severity Index). Se

Revista Methodo: Investigación Aplicada a las Ciencias Biológicas. Facultad de Medicina. Universidad Católica de Córdoba. Jacinto Ríos 571 Bo Gral. Paz. X5004FXS. Córdoba. Argentina. Tel.: (54) 351 4517299 / Correo: methodo@ucc.edu.ar / Web: methodo.ucc.edu.ar| BIOESTADISTICA Y METODOLOGIA APLICADA Rev. Methodo 2020;5(3):108-112. 
trata de un índice con puntuación que tiene en cuenta factores demográficos, las comorbilidades del paciente, datos del examen físico y datos de laboratorio que al sumarlos estiman la mortalidad y el lugar de tratamiento del paciente adulto. De esta forma, un puntaje igual o menor a 70 significa que el paciente tiene un riesgo bajo de mortalidad y puede ser tratado de manera ambulatoria; de 71 a 90 puntos presenta un riesgo más elevado y se recomienda la observación en unidades de corta estancia; de 91 a 130 puntos el riesgo es moderado y se recomienda el ingreso hospitalario; y un puntaje superior a 130 es de alto riesgo y también se debe hospitalizar al paciente ${ }^{5}$.

Los corticoides o corticoesteroides son hormonas esteroideas que se producen en la corteza de las glándulas suprarrenales. Éstas participan en diferentes procesos, regulando la inflamación, el sistema inmunitario, el metabolismo de los hidratos de carbono, metabolismo de proteínas y lípidos, así como la respuesta del organismo frente al estrés. Alguna de sus acciones se ejerce también sobre el sistema nervioso, renal y cardiovascular. Existen dos tipos de corticoides naturales, mineralocorticoides y glucocorticoides. Los corticoides sintéticos y semisintéticos se obtienen mediante la modificación de la estructura química de los naturales; siendo numerosos los procesos patológicos para cuyo tratamiento se utilizan. 6 Todos los esteroides poseen acción primaria sobre el material genético nuclear, estimulando la transcripción. Se unen a receptores específicos, la mayoría localizados en el nucleoplasma, y actúan como un factor de transcripción. Estimula la síntesis de ARNm y de proteínas específicas. Los efectos metabólicos se deben en gran parte a los cambios en la producción de enzimas 6 .

Las principales reacciones adversas de los corticoides son, la redistribución de la grasa corporal, hiperglucemia que puede evolucionar a diabetes, hipertensión arterial, aumento del riesgo de infecciones, osteoporosis, disminución del crecimiento en niños, aumento del riesgo cardiovascular, insuficiencia suprarrenal en caso de retirada brusca del fármaco, riesgo de cataratas y glaucoma, entre otros ${ }^{6}$.

\section{Búsqueda bibliográfica}

Para responder a la pregunta planteada se realizó una búsqueda en PubMed utilizando los siguientes MeSH (Medical Subject Headings): "pneumonia AND glucocorticoids", se obtuvieron el resultado de 1705 artículos. Luego de aplicar los filtros en base al tipo de artículo y la fecha de publicación (revisiones sistemáticas y 5 años, respectivamente) se obtuvieron un resultado de 12 artículos. Luego de la lectura de los títulos y resúmenes de los mismos, se seleccionó: "Efficacy and safety of glucocorticoids in the treatment of severe community-acquired pneumonia"7, el cual se corresponde con un nivel de evidencia Ia.

\section{Resumen del artículo}

El estudio seleccionado se trata de un metaanálisis que tiene como objetivo evaluar la eficacia y seguridad de la terapia adyuvante con glucocorticoides en el tratamiento de la neumonía severa adquirida de la comunidad. Para ello, realizaron una búsqueda en las bases de datos de PubMed, EMBASE, Cochrane Library, EBASO, Medline, Google Scholar, Science Dicet, CBM y CNKI para analizar diversos ensayos aleatorizados controlados hasta enero de 2018.

Obtuvieron un total de 2075 estudios a los cuales se les aplicaron diferentes criterios de exclusión. Finalmente, analizaron un total de 10 ensayos aleatorizados controlados con un total de 665 pacientes, mayores de 18 años con diagnóstico de neumonía adquirida de la comunidad grave, según el score PSI.

Evaluaron un grupo experimental y uno control. Ambos fueron tratados con antibioticoterapia, sin embargo, al grupo experimental se les añadió corticoterapia de manera adyuvante. Se midió como resultados de eficacia todas las causas de mortalidad, los niveles de proteína $\mathrm{C}$ reactiva (PCR), la incidencia de shock séptico y la necesidad de ventilación mecánica. Eventos adversos incluyendo la superinfección, la hemorragia digestiva alta y la hiperglucemia fueron los resultados de seguridad.

En cuanto a los resultados de eficacia, los corticoides adyuvantes parecieron ser superiores en comparación con el tratamiento convencional en términos de mortalidad por cualquier causa (Riesgo Relativo [RR]: 0,47, IC 95\% 0,3-0,74 $\mathrm{p}=0,001)$, los niveles de PCR el día 8 posterior a la administración (Diferencia Media Estándar [DME]: $-0,8$, IC 95\%, $-1,11$ a $-0,5, \mathrm{p}=0,001)$, la incidencia de shock séptico (Odds Ratio [OR] $0,15$, IC 95\% 0,07-0,29, $\mathrm{p}=0,001)$ y el requerimiento de ventilación mecánica (OR: $0,32$, IC $95 \%, 0,20-0,52, p=0,001)$. En cuanto a los resultados de seguridad, no se observaron diferencias entre los 2 grupos en cuanto a la hemorragia digestiva alta (OR: 0,83, IC 95\%, $0,27-2,52, \mathrm{p}=0,74)$, hiperglucemia (OR: 1,3 , IC

Revista Methodo: Investigación Aplicada a las Ciencias Biológicas. Facultad de Medicina. Universidad Católica de Córdoba. Jacinto Ríos 571 Bo Gral. Paz. X5004FXS. Córdoba. Argentina. Tel.: (54) 351 4517299 / Correo: methodo@ucc.edu.ar / Web: methodo.ucc.edu.ar| BIOESTADISTICA Y METODOLOGIA APLICADA Rev. Methodo 2020;5(3):108-112. 
Alegre G, Buffa A, Colussi M Y, Giménez C, Manzanares S, Monferini F, Paschini J. El uso de glucocorticoides en neumonía grave adquirida de la comunidad.

$95 \% 0,68-2,49, \mathrm{p}=0,42)$ y superinfeccion (OR: 1,11, IC $95 \%$ 0,14-9,13, p=0,92).

En el estudio analizado concluyeron que la corticoterapia adyuvante produjo resultados favorables en el tratamiento de la neumonía adquirida de la comunidad grave, demuestra la disminución de la mortalidad, de la incidencia de shock séptico y del requerimiento de ventilación mecánica, sin incrementar el riesgo de efectos adversos.

\section{Análisis del artículo}

Para responder la pregunta clínica estructurada, que pretende determinar si el uso de corticoterapia en la neumonía adquirida de la comunidad grave disminuye la mortalidad y el uso de asistencia respiratoria mecánica, hemos analizado distintas variables estadísticas. Se definieron el riesgo absoluto (RA), la reducción del riesgo absoluto (RRA), reducción del riesgo relativo (RRR) y el número necesario a tratar (NNT) con sus respectivos intervalos de confianza del 95\% (IC 95\%).

Para la variable de mortalidad nos centramos en los valores descritos en el estudio, se realizó el análisis estadístico correspondiente para determinar si existía una diferencia para las distintas opciones de tratamiento. La mortalidad fue del $7 \%$ en los pacientes que recibieron tratamiento adyuvante con corticoides, mientras que fue del $16 \%$ en aquellos que recibieron el tratamiento estándar. Con respecto al RRA, se demostró una reducción del 8.5\% (IC 95\% 3,4 $13,6 \%$ ) de la mortalidad en pacientes que recibieron corticoterapia adyuvante. En relación al RRR, se evidenció una reducción del 53\% (IC $95 \% 21-84 \%$ ) en la mortalidad en aquellos pacientes que recibieron corticoterapia. Finalmente, el NNT fue de 12 (IC 95\% 7- 29), es decir, por cada 12 pacientes tratados con corticoterapia se evitó la muerte de una.

En relación a la segunda variable estudiada, la necesidad de asistencia respiratoria mecánica, se realizó un análisis semejante, a fin de determinar los datos estadísticos. Se observó que la necesidad de asistencia respiratoria mecánica en los pacientes que recibieron corticoterapia adyuvante fue del $13 \%$, frente a los pacientes que recibieron tratamiento estándar, donde la necesidad de ARM fue del $30 \%$. En cuanto al RRA, se demostró una reducción del 17\% (IC 95\% 9,8 - 24,2\%) de necesidad de ARM en los pacientes con corticoterapia. El RRR fue del $56 \%$ (IC 95\% 32 - 80\%) en los pacientes que recibieron tratamiento adyuvante con corticoides frente a los que recibieron el tratamiento estándar. Por último, el NNT fue de 6 (IC 95\% 4
- 10), concluyendo que se necesitan tratar a 6 pacientes con corticoterapia adyuvante para evitar que uno requiera ARM.

\section{Comentarios y recomendaciones finales}

El tratamiento adyuvante con glucocorticoides en la neumonía grave adquirida de la comunidad demostró reducir la mortalidad y el requerimiento de ARM. Debido a la alta disponibilidad de los fármacos, su bajo costo económico y a los beneficios que otorga se recomienda el uso de corticoterapia adyuvante como tratamiento contra la NAC grave.

\section{Bibliografía}

1. Xaubet Mir A. Neumología. En: Farreras P., Rozman C. Medicina Interna. XVIII edición. España: Elsevier; 2016. p. 710722.

2. Lopardo G, Basombrío A, Clara L, Desse $\mathrm{J}$, et al. Neumonia Adquirida de la comunidad en adultos. Recomendaciones sobre su atención. Medicina (Buenos Aires). 2015; 75: 245-257.

3. Giovini V, Aguirre R, Rolando L, Guzmán M, Absi R, Vay C, et al. Neumonía Grave de la Comunidad (NGC), 145 casos internados en terapia intensiva. Rev Am Med Resp. 2009; 9: 181-189.

4. Jain S, Self WH, Wunderink RG, Fakhran S. Community-Acquired Pneumonia Requiring Hospitalization among U.S. Adults. N Engl J Med. 2015 Jul 30;373(5): 415-27.doi: 10.1056/NEJMoa1500245.

5. España P, Capelastegui A. Neumonía adquirida en la comunidad de carácter grave: valoración y predicción. Medicina Respiratoria. 2008; (1) 3:7-17.

6. Blanco Antonio. Sistema endócrino. Química Biológica. VII edición. 2da. reimpresión. Buenos Aires: El Ateneo; 2002. p. 385-446.

7. Jiang S, Liu T, Hu Y, et al. Efficacy and safety of glucocorticoids in the treatment of severe community-acquired pneumonia: A meta-analysis. Medicine (Baltimore). 2019;98(26): e16239. doi:10.1097/MD.0000000000016239.

Revista Methodo: Investigación Aplicada a las Ciencias Biológicas. Facultad de Medicina. Universidad Católica de Córdoba. Jacinto Ríos 571 Bo Gral. Paz. X5004FXS. Córdoba. Argentina. Tel.: (54) 351 4517299 / Correo: methodo@ucc.edu.ar / Web: methodo.ucc.edu.ar| BIOESTADISTICA Y METODOLOGIA APLICADA Rev. Methodo 2020;5(3):108-112. 
Alegre G, Buffa A, Colussi M Y, Giménez C, Manzanares S, Monferini F, Paschini J. El uso de glucocorticoides en neumonía grave adquirida de la comunidad.

Link: https://youtu.be/G4gZZd2DWjE

transmisión del ateneo central Facultad Ciencias de la Salud.

\section{(c) (1)(2)(0) \\ BY NC SA}

Revista Methodo: Investigación Aplicada a las Ciencias Biológicas. Facultad de Medicina. Universidad Católica de Córdoba. Jacinto Ríos 571 Bo Gral. Paz. X5004FXS. Córdoba. Argentina. Tel.: (54) 351 4517299 / Correo: methodo@ucc.edu.ar / Web: methodo.ucc.edu.ar| BIOESTADISTICA Y METODOLOGIA APLICADA Rev. Methodo 2020;5(3):108-112. 